Int. J. Dev. Biol. 51: 123-129 (2007)

doi: $10.1387 / \mathrm{ijdb} .062200 \mathrm{fk}$

Original Article

\title{
Cadherin-6 is required for zebrafish nephrogenesis during early development
}

\author{
FUMITAKA KUBOTA ${ }^{1}$, TOHRU MURAKAMI*,1, KENJI MOGI ${ }^{2}$ and HIROSHI YORIFUJI ${ }^{1}$ \\ ${ }^{1}$ Neuromuscular and Developmental Anatomy and ${ }^{2}$ Stomatology and Maxillofacial Surgery \\ Gunma University Graduate School of Medicine, Gunma, Japan
}

\begin{abstract}
We performed functional analyses of cadherin-6 (cdh6) in zebrafish nephrogenesis using antisense Morpholino oligonucleotide (MO) inhibition combined with in situ hybridization. We have cloned a zebrafish homolog (accession number AB193290) of human K-cadherin (CDH6), which showed $60-63 \%$ identity and $76-78 \%$ similarity to the human, mouse, chicken and Xenopus homologs. Whole-mount in situ hybridization showed that $c d h 6$ is expressed in the pronephric ducts and nephron primordia in addition to the central and peripheral nervous systems. Expression of $c d h 6$ in the pronephric ducts was first detected at 14 hours post-fertilization (hpf) and increased to $24 \mathrm{hpf}$. Embryos injected with MOs directed against cdh6 (cdh6MOs) showed developmental defects, including a small head, body axis curvature, short yolk extension and a short bent tail by $30 \mathrm{hpf}$ and edema appeared in the thorax by $42 \mathrm{hpf}$. Such defects and edema became more marked by $\mathbf{5 2}$ hpf and most of the affected embryos died by 5 days post-fertilization. Embryos injected with cdh6MOs were subjected to in situ hybridization with probes for the pronephric markers, wt1 and pax2.1, to examine disturbed development of the anterior region of the pronephric ducts and the nephron primordia. Histological studies showed malformation of the pronephros as abnormally fused glomerulus primordia, fused or abnormally bent pronephric tubule anlagen and coarctated pronephric ducts. These results suggest that cdh6 plays pivotal roles in the development of the pronephros in zebrafish embryos.
\end{abstract}

KEY WORDS: cadherin-6, K-cadherin, zebrafish, Morpholino, pronephros

\section{Introduction}

Cell-cell interaction is a fundamental process required for development (Hynes and Lander, 1992). The cadherins are cellsurface molecules that mediate cell-cell adhesion mainly through homophilic interactions (Takeichi, 1991). Most cadherins exhibit unique expression patterns and functional studies of several cadherins have shown that these molecules play essential roles in the development of vertebrate tissues and organs (Yagi and Takeichi, 2000).

There have been a number of studies of the expression and function of cadherin- 6 (K-cadherin), a member of the classical type II cadherin subfamily (Redies, 1995), in normal renal development (Cho et al., 1998; Mah et al., 2000) and in the formation of renal carcinoma (Xiang et al., 1994). Cadherin- 6 has also been shown to be expressed in the mouse, chicken and Xenopus nervous systems (Inoue et al., 1997; Nakagawa and Takeichi, 1998; David and Wedlich, 2000). Liu et al. recently reported the expression of cadherin- 6 in the nervous system and pronephric ducts of the zebrafish (Liu et al., 2006). However, they presented only a brief description of cadherin- 6 expression in the zebrafish pronephros and there have been no reports regarding its function.

The zebrafish pronephros consists of a single glomerulus, a pronephric tubule and a pronephric duct. The pronephric primordium is first evident during early somitogenesis as a mass of intermediate mesoderm underlying the second and third somites (Kimmel et al., 1995). Growth and differentiation of the pronephric ducts follow behind somitogenesis and are completed by 24 hours post-fertilization (hpf), by which time nephron primordia have formed at the anterior tips of the pronephric ducts (Drummond et al., 1998).

The zebrafish pronephros, although more primitive than the differentiated metanephros, shares many features with this type

Abbreviations used in this paper: CDH, cadherin; hpf, hours post-fertilization; ISH, in situ hybridization; MO, Morpholino oligonucleotide.

\footnotetext{
*Address correspondence to: Dr. Tohru Murakami. Neuromuscular and Developmental Anatomy, Gunma University Graduate School of Medicine, 39-22, Showa-machi 3-chome, Maebashi, Gunma 371-8511, Japan. Fax: +81-27-220-7916. e-mail: mura@med.gunma-u.ac.jp
} 
of kidney. For example, similar to the metanephros, the pronephros has a glomerulus lined with podocytes with foot processes that form the glomerular basement membrane together with endothelia (Drummond et al., 1998). In addition, the tubules of the pronephros have a brush-border similar in appearance to that in the polarized epithelium of the metanephric kidney (Drummond et al., 1998; Drummond, 2000).

Therefore, the zebrafish pronephros represents a valid model for the development of more complex vertebrate kidneys. The inductive events leading to the formation of the pronephros and mesonephros are thought to be quite similar to those directing formation of the metanephros (Vize etal., 1997). In fact, a number of genes involved in metanephric development are also expressed in the zebrafish pronephros: wt1 and pax2.1 are also expressed in the developing glomerulus, tubules and ducts of the pronephros (Kreidberg et al., 1993; Torres et al., 1995; Drummond et al., 1998; Drummond, 2000).

Many types of cadherin appear to be important for kidney morphogenesis. In zebrafish, cadherin17 (cdh17) is expressed specifically in the posterior portion of the pronephric ducts during embryonic development and knockdown of cdh17disrupts the normal formation of the posterior portion of the pronephric ducts (Horsfield et al., 2002). We have isolated a zebrafish cadherin that is orthologous to human K-cadherin (CDH6), which we characterized as zebrafish cadherin-6 (cdhb). Here, we report the results of functional analysis of cdh 6 in zebrafish embryos especially in the pronephros and our findings indicating that it plays an essential role in the development of the pronephros.

\section{Results}

\section{Zebrafish cdh6 expressed in the pronephros}

In addition to its expression in the central and peripheral nervous systems, zebrafish cdh6(DDBJ no. AB193290) was expressed in the pronephros during the early stages of development. We analyzed the expression pattern of $c d h 6$ in pronephric regions by whole-mount in situhybridization at 24 , 28 and $30 \mathrm{hpf}$ (Fig. 1). At $24 \mathrm{hpf}$, the zebrafish pronephros consists of pronephric ducts and nephron primordia. About $4 \mathrm{~h}$ later, at $\sim 28 \mathrm{hpf}$, the nephron primordia of both sides come into close

Fig. 1. $c d h 6$ is expressed in the pronephros during early embryogenesis. Expression patterns in pronephric regions were determined by ISH at $24(A-D), 28(E-H)$ and $30(\mathrm{I}-\mathrm{N})$ hpf. (A,E,I) Dorsal views of whole-mount embryos with rostral toward the top. $(\mathbf{B}, \mathbf{F}, \mathbf{J})$ Ventral views around the pronephric region with rostral toward the top. (C, $, \mathbf{G}, \mathbf{K})$ Transverse sections of embryos at the nephron primordium region (Section level is indicated by (1) in the schematic illustration below). (D, $\mathbf{H}, \mathbf{L})$ Transverse sections of embryos at the pronephric duct region (Section level (2) in the illustration below). (M,N) Transverse histological sections $6 \mu \mathrm{m}$ thick at the same regions as Fig. $2 \mathrm{~K}$ and L, respectively. Open white arrowheads, pronephros; open black arrowheads, nephron primordia; open white arrows, glomerular primordia; white arrows, tubule primordia; black arrows, anterior region of the pronephric ducts; closed white arrowheads, organ primordia of the digestive system; closed black arrowheads, dorsal spinal cord. Illustrations at 24, 28 and 30 hpf indicate cdh6 expression (light blue, weak expression): (a) glomerular primordium, (b) tubule primordium, (c) anterior region of the pronephric duct (at 24 hpf, (a) and (b) indicate nephron primordium). Scale bars, $30 \mu \mathrm{m}$.
TABLE 1

MALFORMATIONS INDUCED BY CDH6 ANTISENSE MORPHOLINO OLIGONUCLEOTIDES (CDH6MOS)

$\mathrm{n}$ : Number of embryos counted as viable at $6 \mathrm{hpf}$. Success of injections was determined by monitoring fluorescent labeling of the Morpholino

\begin{tabular}{|c|c|c|c|c|}
\hline Morpholino & $\begin{array}{c}\text { Developmental } \\
\text { defects at } 30 \mathrm{hpf}\end{array}$ & $\begin{array}{l}\text { Edematous embryos } \\
\text { at } 52 \mathrm{hpf}\end{array}$ & $\begin{array}{l}\text { Survival of embryos } \\
\text { until } 5 \mathrm{dpf}\end{array}$ & $\mathbf{n}$ \\
\hline cdhoMO-1 $(2 \mathrm{mg} / \mathrm{ml})$ & $70.5 \%$ & $71.5 \%$ & $27.0 \%$ & 200 \\
\hline$(1 \mathrm{mg} / \mathrm{ml})$ & $33.9 \%$ & $36.6 \%$ & $61.6 \%$ & 112 \\
\hline cdhGMO-2 (2 mg/ml) & $50.1 \%$ & $53.9 \%$ & $43.8 \%$ & 89 \\
\hline$(1 \mathrm{mg} / \mathrm{ml})$ & $20.5 \%$ & $20.5 \%$ & $78.2 \%$ & 78 \\
\hline \multicolumn{5}{|l|}{ Negative } \\
\hline control $\mathrm{MO}(2 \mathrm{mg} / \mathrm{ml})$ & $0.0 \%$ & $0.0 \%$ & $94.0 \%$ & 200 \\
\hline$(1 \mathrm{mg} / \mathrm{ml})$ & $0.0 \%$ & $0.0 \%$ & $95.0 \%$ & 100 \\
\hline
\end{tabular}
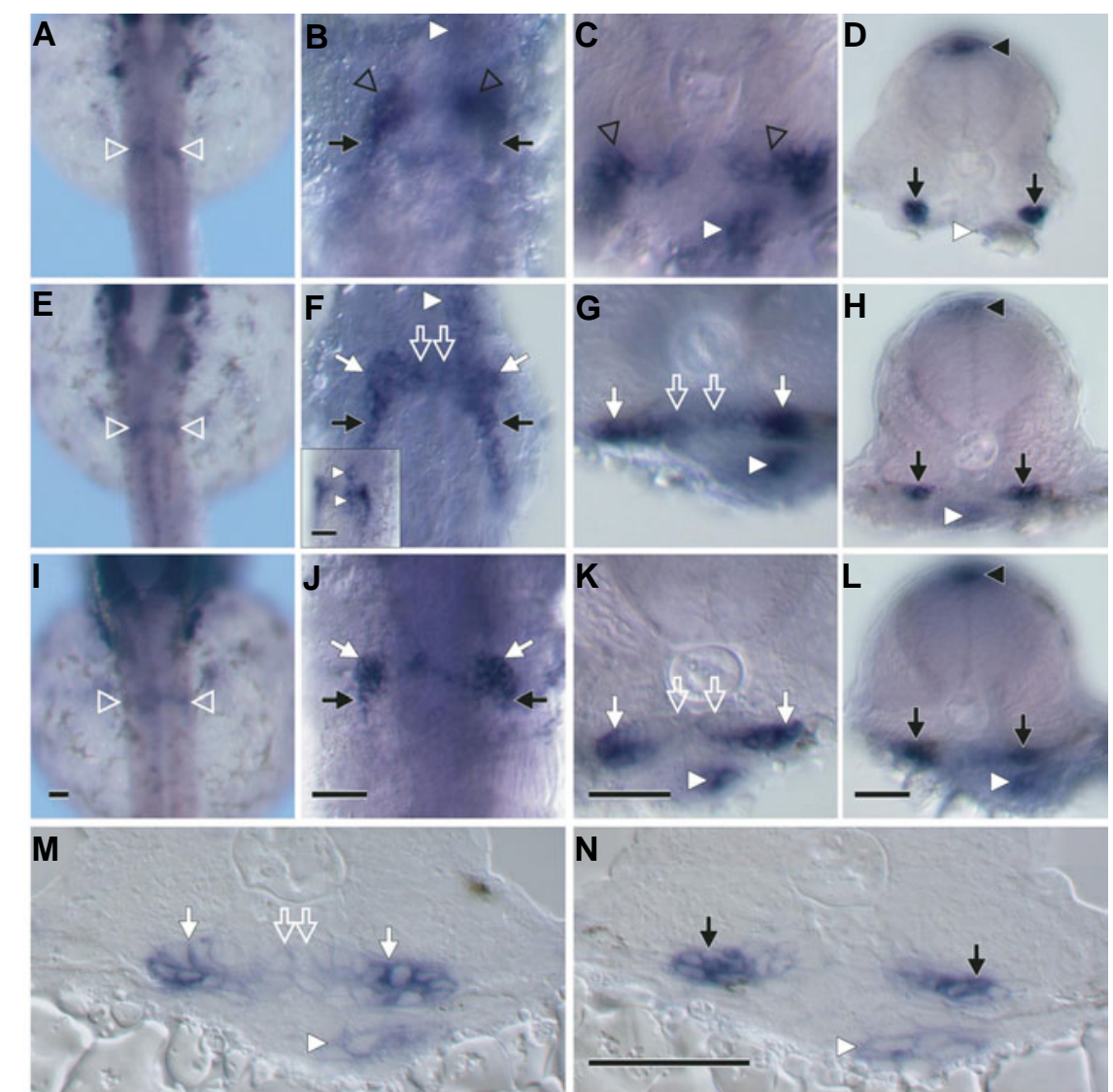

(a)
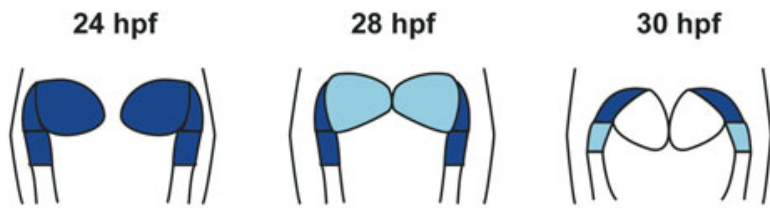

\footnotetext{
anterior region of the pronephric duct (at 24 hpf, (a) and (b) indicate nephron primordium). Scale bars, $30 \mu \mathrm{m}$.
} 
contact and differentiate into the tubular and glomerular primordia.

By $24 \mathrm{hpf}$, cdh 6 was detected in the anterior region of the pronephric duct and nephron primordium (Fig. 1A-D). By $28 \mathrm{hpf}$, cdh6 expression in the glomerular primordium became weaker than in the other positive regions and was not found at $30 \mathrm{hpf}$ (Fig. $1 \mathrm{E}-\mathrm{L})$. Expression of $c d h 6$ was detected in the tubule primordium until $30 \mathrm{hpf}$ (Fig. 1E-L). Its expression was also noted in the pronephric duct around $28 \mathrm{hpf}$, which became weaker by $30 \mathrm{hpf}$ (Fig. 1E-L).

To determine the arrangement of cdh6-positive cells in detail, frozen sections of 30-hpf embryos stained for cdh6were made for histological studies (Fig. 1M, N). Cells showing positive staining for cdh6were found clearly in the pronephric tubule primordia and pronephric duct (Fig. 1M, N). In addition, cdh6 expression was detected in the organ primordia of the digestive system (closed white arrowheads in Fig. 1B-D, $\mathrm{F}-\mathrm{H}, \mathrm{K}-\mathrm{N}$ ) and in the dorsal spinal cord (closed black arrowheads in Fig. 1D, H, L) throughout the experimental period.

\section{Zebrafish cdh6 antisense Morpholino oligonucleotides in- duced embryo malformation}

To determine the role of $c d h 6$, we used two different antisense Morpholino oligonucleotides (MOs) complementary to the translational initiation site of $c d h 6, c d h 6 \mathrm{MO}-1$ and cdhoMO-2, to induce targeted knockdown of cdh6function. Briefly, aliquots of about $8 \mathrm{nl}$ of cdh6MOs were microinjected into embryos at the 1-cell stage at a concentration of 1 or $2 \mathrm{mg} / \mathrm{ml}$. At $6 \mathrm{hpf}$, MO-injected embryos were examined for viability and for fluorescence labeling corresponding to Morpholino distributed throughout the embryos. To determine the effects of cdhoMOs, only those fulfilling both of these conditions were used for further experiments.

By 24 hpf, embryos injected with cdh6MOs showed developmental defects, including small head, small eyes, abnormal body axis curvature, short yolk extension and a short bent tail. These abnormal phenotypes were obvious by 30 hpf (Table 1; Fig. 2C, E).

As cdh17-knockdown embryos showed a disturbance in the cloaca region (Horsfield et al., 2002), we also examined the phenotype of the cloaca in whole fish. No abnormalities were ob-
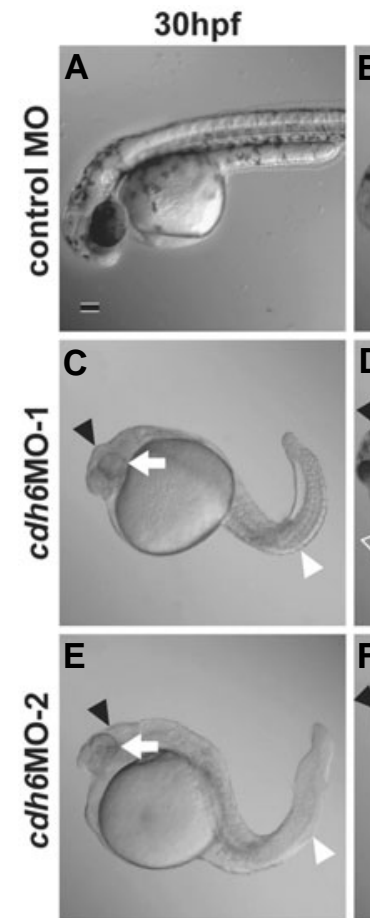

TABLE 2

\section{PRONEPHROS DISTURBED BY CDH6 ANTISENSE MORPHOLINO OLIGONUCLEOTIDES (CDHGMOS)}

\begin{tabular}{llr} 
Injection & Phenotypes revealed by ISH & $\mathbf{n}$ \\
\hline No injection & $\mathrm{N}: 100 \%$ & 30 \\
Negative control Morpholino & $\mathrm{N}: 100 \%$ & 50 \\
cdh6MO-1 & $\mathrm{F}: 16.0 \% \mathrm{CV}: 20.8 \%$ & 106 \\
& $\mathrm{CA}: 12.3 \% \mathrm{~N}: 58.5 \%$ & \\
cdh6MO-2 & $\mathrm{F}: 10.9 \% \mathrm{CV}: 16.3 \%$ & \\
& $\mathrm{CA}: 6.5 \% \mathrm{~N}: 79.3 \%$ & 68 \\
cdh6MO-1 + cdh6mRNA & $\mathrm{F}: 2.9 \% \mathrm{CV}: 4.4 \%$ & \\
cdh6MO-2 + cdh6mRNA & $\mathrm{CA}: 1.5 \% \mathrm{~N}: 94.1 \%$ & 56 \\
\hline
\end{tabular}

Abbreviations: $\mathrm{N}$, almost normal; $\mathrm{F}$, both pronephric tubule primordia fused; $\mathrm{CV}$, pronephric tubule primordia curved abnormally; CA, coarctated pronephric ducts

$\mathrm{n}$ : Number of embryos counted as viable at 6 hpf. Success of injections was determined by monitoring fluorescent labeling of the Morpholino.

served in the posterior portion of the pronephric duct or cloaca in either control or cdhoMO-injected embryos at 30 or 52 hpf (Fig. $2 \mathrm{H}, \mathrm{J}, \mathrm{L}, \mathrm{N})$.

Following the abnormal development in cohoMO-injected embryos, areas of edema appeared in the thorax around $42 \mathrm{hpf}$,
$52 \mathrm{hpf}$
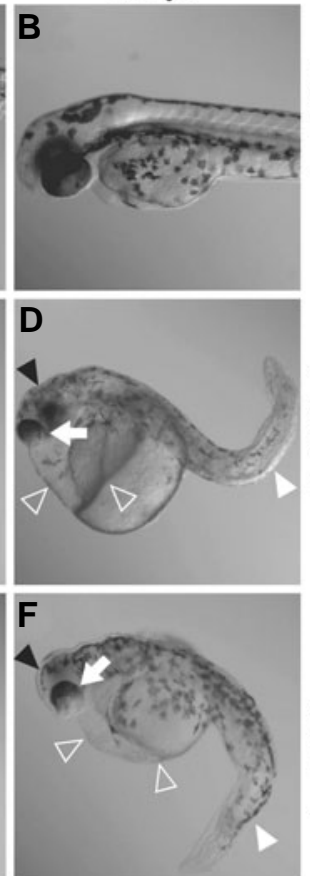

Fig. 2. Zebrafish $\boldsymbol{c d h} 6$ is necessary for normal development. Malformations were detected in zebrafish injected with a cdh6 antisense Morpholino oligonucleotide (cdh6MO-1 or cdh6MO2). (A, $\mathbf{B}, \mathbf{G}, \mathbf{H}, \mathbf{K}, \mathbf{L})$ Embryos injected with control MO. (C, $\mathbf{D}, \mathbf{I}, \mathbf{J}, \mathbf{M}, \mathbf{N})$ Embryos injected with cdh6MO-1. (E,F) Embryos injected with cdh6MO-2. (A,C,E, G-J), 30 hpf. (B,D,F, K-N), 52 hpf. (AF) Lateral views of whole-mount specimens with rostral to the left. $(\mathbf{G}, \mathbf{I}, \mathbf{K}, \mathbf{M})$ Higher magnification views of head regions. $(\mathbf{H}, \mathbf{J}, \mathbf{L}, \mathbf{N})$ Higher magnification views of cloaca regions. Black arrowheads, small head; white arrows, small eye; closed white arrows, bent tail; black arrows, posterior portion of the pronephric duct and cloaca; open white arrows, short yolk extension; open white arrowheads, edema in the thorax. Concentrations of cdh6MOs and control MO were $2 \mathrm{mg} / \mathrm{ml}$. Scale bars, $200 \mu \mathrm{m}$.
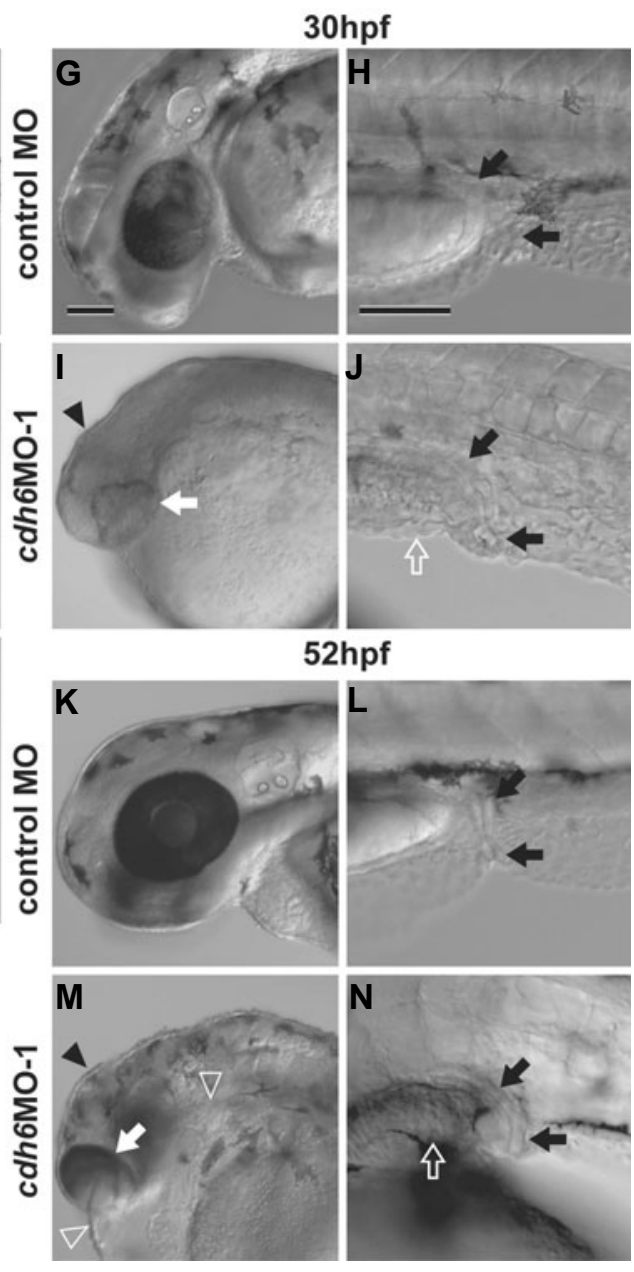
which became more marked at 52 hpf (Table 1; Fig. 2D, F, M). Embryos injected with cdhoMO-1 or cdh6MO-2 showed virtually the same phenotypes and similar percentages of abnormalities at 30 and 52 hpf (Table 1). Most of the embryos with MO injections died by 5 days post-fertilization (dpf) (Table 1).

\section{Zebrafish cdh6 antisense morpholino oligonucleotides dis- rupted normal nephrogenesis}

To investigate the disturbed regions in detail, the colhoMOsinjected embryos were subjected to in situ hybridization with probes for the pronephric markers, wt1 and pax2.1.

$w t 1$ is expressed in glomerular primordia and is limited to the podocytes in mature fish (Drummond et al., 1998). In the present study, wt1 was expressed in the glomerular primordia at $30 \mathrm{hpf}$ in embryos injected with control MO (Fig. 3A). Both of the glomerular anlagen were symmetrical and showed a contacted arrangement. In contrast, the cdhoMO-injected embryos showed quite different phenotypes; in some cases, glomerular primordia of both sides showed abnormal fusion (Fig. 3B), while in others the glomerular primordium showed asymmetric expression and localization (Fig. 3C).

For quantitative evaluation of the abnormalities in pronephros development, we also stained for pax2. 1, which is known to be expressed in the pronephric tubule primordia and the pronephric

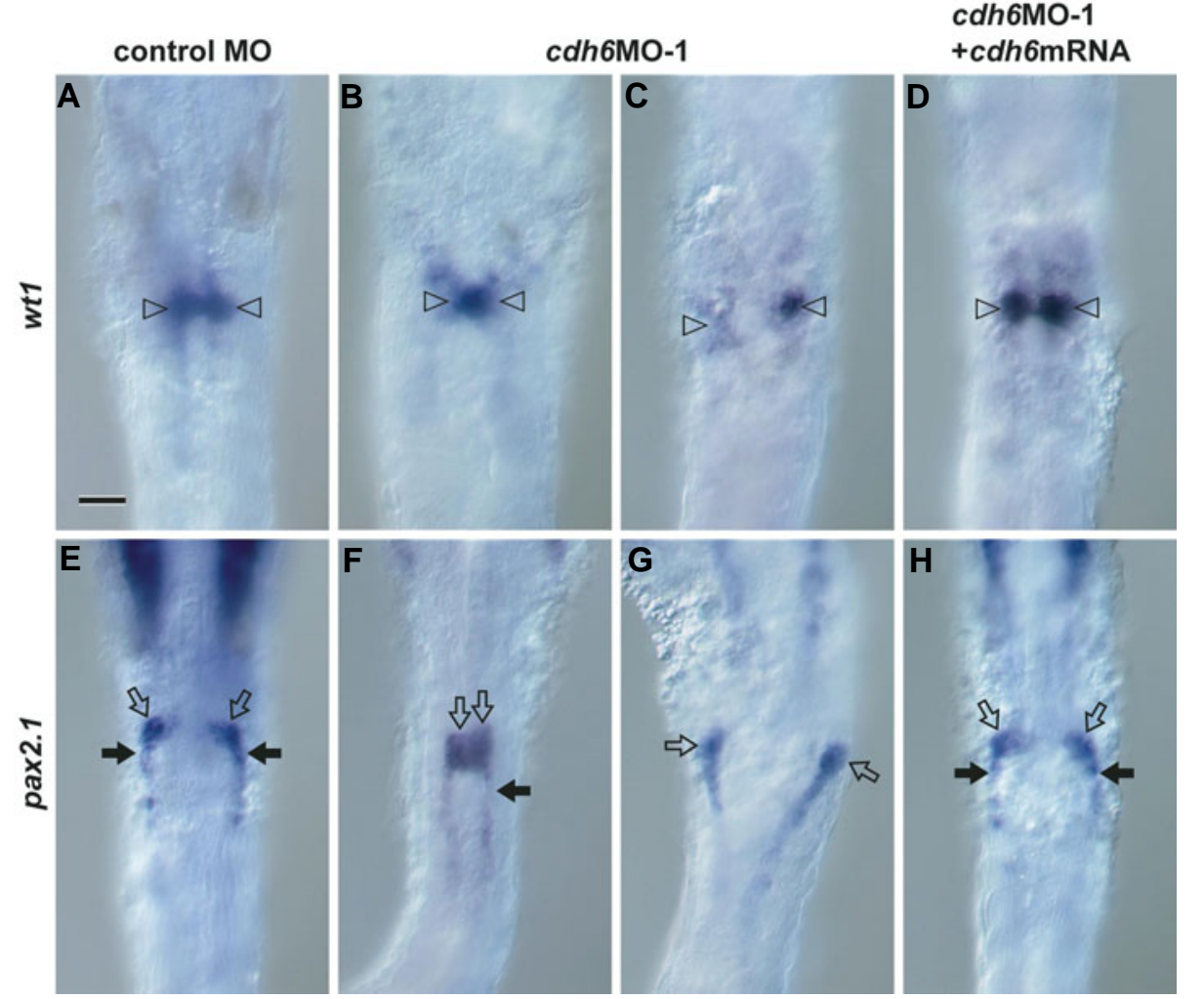

Fig. 3. Expression of $\boldsymbol{c d h} \mathbf{6}$ is required for normal pronephros formation. $(A-H)$, Ventral views of the pronephric regions with rostral at the top at $30 \mathrm{hpf}$. (A,E) Embryos injected with control MO. (B,C,F,G) Embryos injected with cdh6 antisense MO (cdh6MO-1). (D,H) Embryos injected with cdh6MO-1 and cdh6mRNA. (A-D) Stained by in situ hybridization for wt1 to show glomerular primordia. Open arrowheads, glomerular primordia. (E-H) Stained for pax2.1 to show tubule primordia and pronephric ducts. Open arrows, tubule primordia; closed arrows, pronephric ducts. Concentrations of cdh6MO-1 and control MO were $1 \mathrm{mg} / \mathrm{ml}$. Scale bar, $50 \mu \mathrm{m}$. ducts (Drummond etal., 1998) (Fig. 3E). At $30 \mathrm{hpf}$, the expression patterns of embryos injected with cdhoMO-1 or cah6MO-2 could be classified into four types: (1) expression in the pronephros resembling that in negative controls (class $N$ ), (2) both pronephric tubule primordia fused (class F) (Fig. 3F), (3) either or both of the pronephric tubule primordia curved abnormally (class CV) (Fig. $3 \mathrm{G}$ ) or (4) coarctation found in the pronephric ducts (class CA) (Fig. 3F). Embryos showing two expression phenotypes (as in Fig. 3F) were counted once for each phenotype. The results were as follows: cdhoMO-1; F 16.0\% CV 20.8\% CA 12.3\% N 58.5\%. cdhoMO-2; F 10.9\% CV 16.3\% CA 6.5\% N 79.3\% (Table 2). To confirm specificity of the cdhoMOs, we performed RNA rescue experiments using cah6 mRNA; 94-95\% of the RNA-rescued embryos showed no abnormalities throughout the whole body and were categorized as showing class $\mathrm{N}$ phenotype (Table 2; Fig. 3D, H). As the percentages of affected embryos at 30 and 52 hpf were almost the same, we stained injected embryos for pax2.1 at $30 \mathrm{hpf}$.

In addition to the investigation at the organ level, we studied the effects of knockdown of cdh6 expression on the cells. The cell arrangement in cdh6-positive regions of the pronephros was examined in frozen sections (Fig. 4). Epithelial cells of glomerular primordia, tubule primordia and pronephric duct regions in control embryos showed well-ordered cellular arrangements (Fig. 4AC). However, the epithelial cells in each region of the embryos affected by cdh6MO-1 showed a disturbed appearance. The cells in the pronephros were disorganized and had an abnormal structure (Fig. 4D-F).

\section{Discussion}

cdh6 is expressed in the glomerular and pronephric tubule primordia and the anterior region of the pronephric ducts

Zebrafish cdh6 was expressed in the glomerular and pronephric tubule primordia and in the anterior region of the pronephric ducts. We examined the expression pattern of $c d h 6$ in the pronephros in detail. During zebrafish nephrogenesis, $c d h 6$ was expressed in nephron primordia and in the anterior region of the pronephric ducts from $14 \mathrm{hpf}$, at the beginning of nephrogenesis, to $24 \mathrm{hpf}$. From 24 to $30 \mathrm{hpf}$, cdh6 was expressed in the glomerular and the pronephric tubule primordia and in the anterior region of the pronephric ducts. In zebrafish, the stages from 24 to $36 \mathrm{hpf}$ are important for proper pronephros development, as normal nephron primordia differentiate into glomerular and pronephric tubule primordia during this stage (Drummond et al., 1998).

Cadherin- 6 is expressed in the developing nervous system and kidney in mammals and has been suggested to play important roles in the formation of epithe- 
Fig. 4. Epithelial cells of affected embryos were deformed by cdh6 MOs. (A-F), Transverse histological sections of $5 \mu \mathrm{m}$ thick at 30 hpf. (A-C) Embryos injected with control MO. (DF) Embryos injected with cdh6 antisense MO (cdh6MO-1). (A,D) Stained by in situ hybridization for wt1 to show glomerular primordia. (B,C,E,F) Stained for pax2.1 to show tubule primordia and pronephric ducts. Open white arrowheads, glomerular primordia; open white arrows, tubule primordia; closed black arrows, pronephric ducts; closed white arrows, dorsal aorta; $N$, notochord. Concentrations of cdh6MO1 and control MO were 1 $\mathrm{mg} / \mathrm{ml}$. Scale bar, $20 \mu \mathrm{m}$.

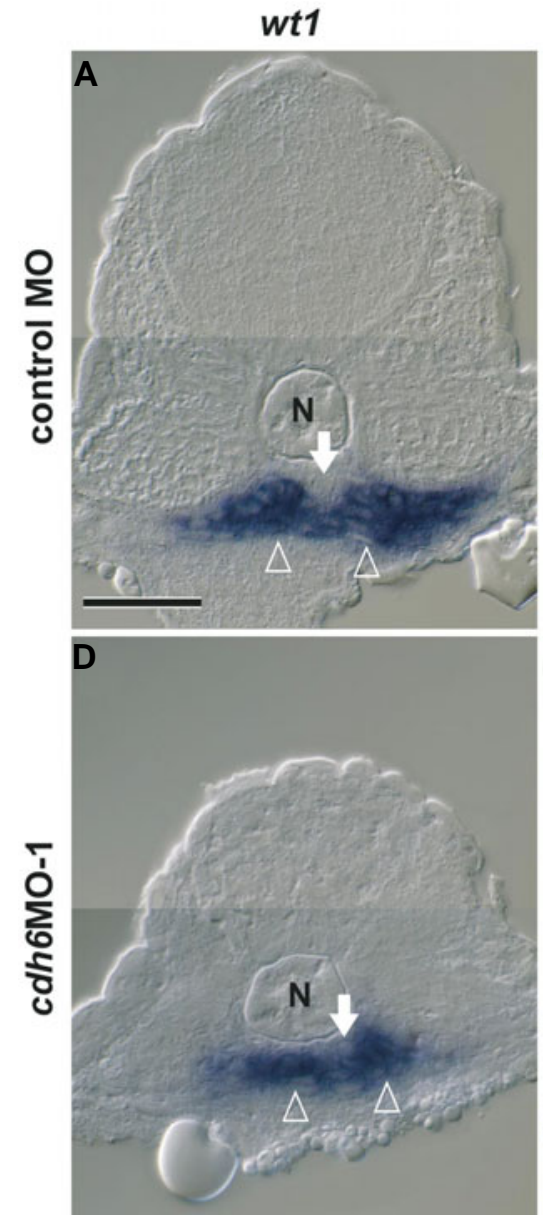

lial cells in the latter (Cho et al., 1998; Inoue et al., 1998).

Zebrafish cdh6 is important for the proper formation of the glomerulus, the pronephric tubules and the anterior region of the pronephric ducts during early embryogenesis

Based on the following observations, we concluded that zebrafish cdh6is required for correct formation of the glomerulus, the pronephric tubules and the anterior portion of the pronephric ducts during early embryogenesis.

First, we observed abnormal pronephra in cdh6-knockdown zebrafish embryos. That is, whole-mount specimens showed fused or abnormally curved pronephric tubule primordia, fused abnormal glomerular primordia and coarctated pronephric ducts. Examination of histological sections revealed disturbance of the epithelial tissue; the glomerular and pronephric tubule primordia, as well as the anterior region of the pronephric ducts, were disrupted and the epithelial cells showed a disordered arrangement.

Second, cdh6-knockdown embryos showed obvious edema in the thorax at $52 \mathrm{hpf}$. Filtration at the pronephra is thought to begin between 40 and $48 \mathrm{hpf}$ and the edema observed in these embryos was thought to be caused by abnormal filtration (Drummond etal., 1998). Pronephric mutants have been reported to develop kidney cysts and edema (Drummond et al., 1998; Horsfield et al., 2002). We consider that abnormalities in filtration would be lethal in zebrafish, which have only a single nephron. Therefore, in con-

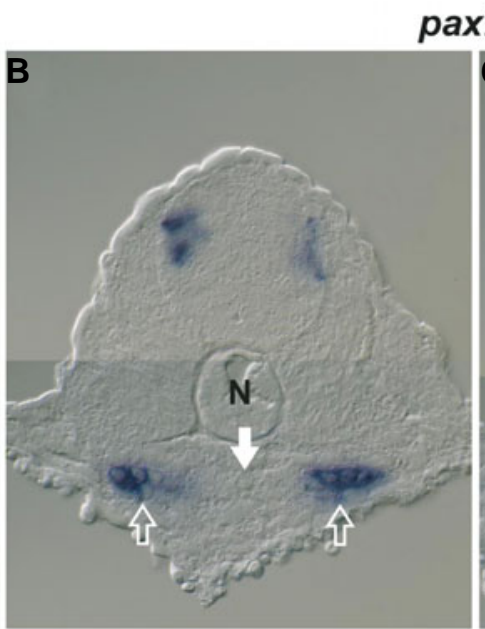

pax2.1
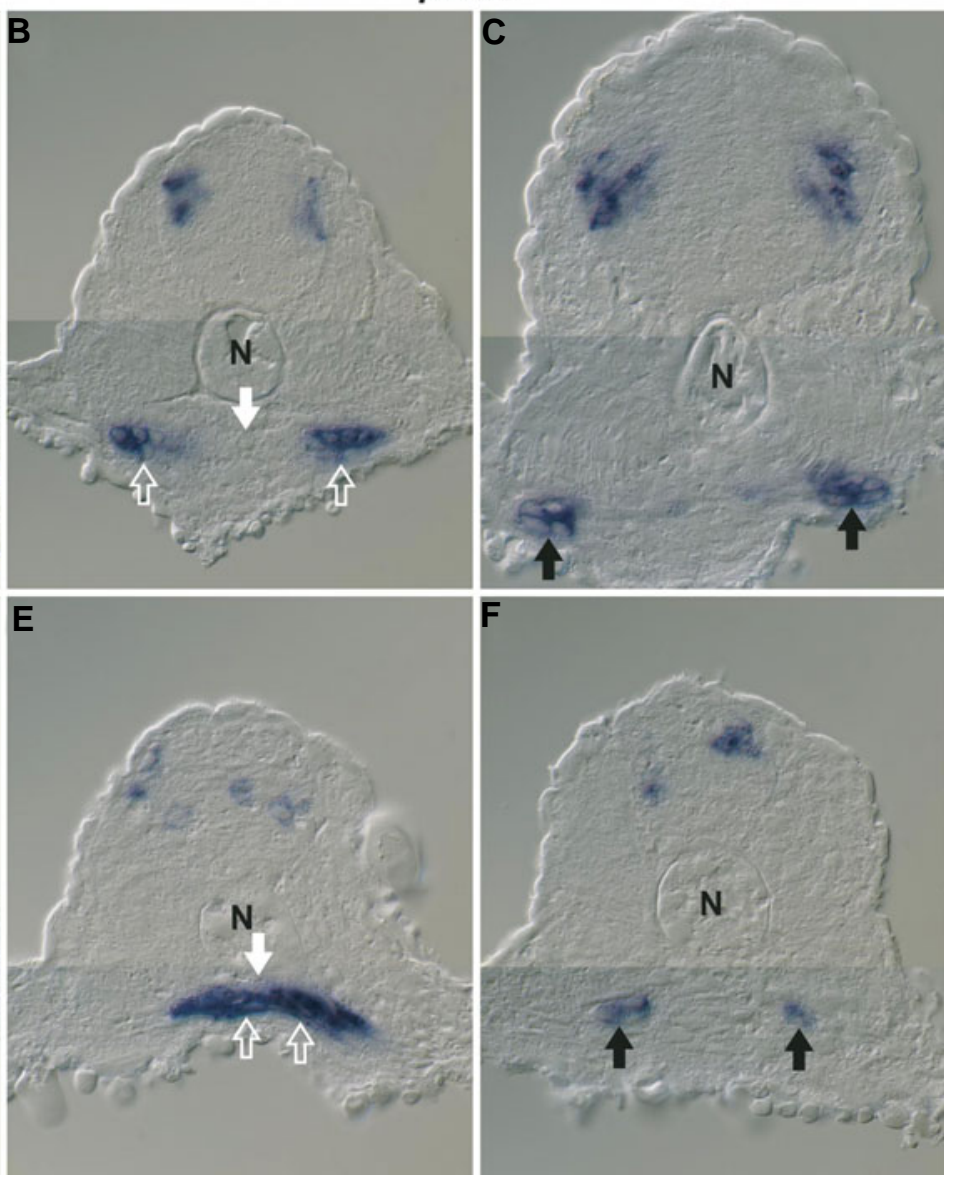

trast to CDH6-knockout mice (Mah et al., 2000), which are both viable and fertile, cdh 6 is necessary for the normal development and survival of zebrafish.

cdh6 and cdh17 seem to regulate development of the different parts of the pronephros during early embryogenesis

During development, $c d h 6$ and $c d h 17$ are expressed in different regions of the pronephros, suggesting that these proteins have mutually supplementary roles in the morphogenesis of the pronephros. While cdh 6 is expressed in the nephron primordia and the anterior portion of the pronephric ducts, chd17 is expressed in the posterior portion of the pronephric ducts in the pronephros. On the other hand, the transcription factors $w t 1$, pax2. 1 and $\operatorname{sim} 1$ are thought to have regulatory effects specific to different parts of the zebrafish kidney (Serluca and Fishman, 2001). These factors seem to have some relationships to the cadherins. The spatial and temporal relationship between sim1 and $c d h 17$ expression-with the timing of sim1 expression being slightly ahead of that of $c d h 17$-suggests that $\operatorname{sim} 1$ may regulate the expression of $c d h 17$ (Horsfield et al., 2002). On the other hand, $c d h 6$ is expressed in the anterior region of the pronephros, which is positive for $w t 1$ and pax2. 1 in early embryogenesis and cdh6-knockdown mutants showed malformation in this area. Although these genes are first expressed in the intermediate mesoderm (Drummond et al., 1998), it is possible that $w t 1$ and/or pax2. 1 act upstream or downstream of $c d h 6$. Further studies are 
necessary to determine the factors involved in regulation of $c d h 6$ and $c d h 17$ expression during early nephrogenesis.

\section{Materials and Methods}

\section{Care of fish and obtaining zebrafish embryos}

Fish were purchased from a local pet shop and used as "wild-type." These wild-type zebrafish were maintained and the embryos were obtained as described previously (Westerfield, 1995). They were staged in hours post-fertilization (hpf) at the standard temperature of $28.5^{\circ} \mathrm{C}$. With the exception of the antisense Morpholino experiment, embryos for whole-mount in situ hybridization were raised from $18 \mathrm{hpf}$ throughout development in 0.003\% 1-phenyl-2-thiourea (PTU; Wako Pure Chemical Ind., Osaka, Japan) to reduce pigmentation.

\section{Cloning of zebrafish genes}

We screened a zebrafish cDNA library for molecules with homology to cadherin, which yielded a number of cadherin and protocadherin genes including cdh6 (Murakami et al., 2006). Zebrafish wt1 and pax2.1 (GenBank: wt1, AY028627; pax2.1, AF067534) were cloned by PCR using a zebrafish cDNA library and specific primers:

wt1 forward ATTTGCTCTGCTCCTGAAAGTCCTC reverse GGAAACACAGTTGTTTATTGGCACC

pax2.1 forward GCTTGCGGTCCCTTAAATATGTAGC reverse CAGGAAATAGTTCAGTGATGGTGCC

\section{In situ hybridization (ISH)}

Digoxigenin-labeled RNA probes were synthesized using the cDNA clones as templates. Staged zebrafish embryos were stained by wholemount ISH as described previously (Weinberg et al., 1996; Bellipanni et al., 2000).

\section{Microinjection of cadherin-6 morpholinos and mRNA}

Fluorescein-labeled antisense Morpholino oligonucleotides (MOs) were purchased from Gene Tools (Philomath, OR, USA). These MOs were designed complementary to the 5' sequence near the translational initiation site of cdh6 (cdhGMOs) with the sequences: cdhoMO-1, 5'AAGAAGTACAATCCAAGTCCTCATC-3' (targets 5' sequence spanning the start codon, italicized); and coh6MO-2, 5'ATCCTATCTGCCAAAGTTACAGAGC-3' (directed against the sequence 5 ' of the UTR to the start codon). The negative control $\mathrm{MO}$ had the sequence: 5'-CCTCTTACCTCAGTTACAATTTATA-3'. Capped cdh6 mRNA was synthesized from cdh6 CDNA subcloned in the pCS2+ vector as described previously (Bellipanni et al., 2000).

cdh6MO alone ( 1 or $2 \mathrm{mg} / \mathrm{ml}$ ) or with cdh6 mRNA $(75 \mu \mathrm{g} / \mathrm{ml})$ were injected into the blastomere of 1-cell embryos or into the yolk using an IM300 microinjector (Narishige, Tokyo, Japan). Success of injection was determined by monitoring GFP fluorescence labeling of the Morpholino.

\section{Histological sections}

Zebrafish embryos stained by ISH for cdh6, wt1 and pax2.1 were embedded in OCT compound (Miles Inc. Diagnostic Division, Elkhart, IN, USA) and snap-frozen in liquid nitrogen. Sections 5 or $6 \mu \mathrm{m}$ thick were cut and mounted on glass slides for microscopy. In some cases, sections of 1-2 somite-thick were cut using a razor blade without freezing.

\section{Microscopy and image processing}

Embryos were mounted on $1.5 \%$ agarose plates with pits cast with 0.5 $\mathrm{mm}$ glass beads and viewed under an Olympus SZX-12 dissection microscope. A Nikon E-1000 compound microscope was used for examination of dissected embryos and histological sections.

Microscopic images were recorded with a C5810 chilled 3CCD camera (Hamamatsu Photonics, Shizuoka, Japan), a Spot RT SE6 Monochrome cooled CCD camera (Diagnostic Instruments, Sterling Heights,
MI, USA) or Olympus E-330 digital SLR camera and processed with Adobe Photoshop on a Macintosh personal computer to match colors to the real images. "Extended-focus" pictures were composed for thick specimens of whole- and flat-mount embryos by taking a series of 2-5 images of the specimen by shifting the focus manually. Each image was placed as an individual layer on a single Photoshop image. Out-of-focus areas in each layer were masked out to reveal the regions of interest of the specimen in-focus in the Photoshop image.

\section{Acknowledgments}

We thank Dr. Hideomi Tanaka and Dr. Hitoshi Okamoto at RIKEN for expert zebrafish husbandry and for their valuable advice. This research was supported in part by Grants-in-Aid awarded to T.M. from the Ministry of Education, Culture, Sports, Science and Technology of Japan (MEXT) and Initiative for Attractive Education in Graduate Schools from MEXT.

\section{References}

BELLIPANNI, G., MURAKAMI, T., DOERRE, O.G., ANDERMANN, P. and WEINBERG, E.S. (2000). Expression of Otx homeodomain proteins induces cell aggregation in developing zebrafish embryos. Dev. Biol. 223: 339-353.

CHO, E.A., PATTERSON, L.T., BROOKHISER, W.T., MAH, S., KINTNER, C. and DRESSLER, G.R. (1998). Differential expression and function of cadherin-6 during renal epithelium development. Development 125: 803-812.

DAVID, R. and WEDLICH, D. (2000). Xenopus cadherin-6 is expressed in the central and peripheral nervous system and in neurogenic placodes. Mech. Dev. 97: 187-190.

DRUMMOND, I.A. (2000). The zebrafish pronephros: A genetic system for studies of kidney development. Pediatr. Nephrol. 14: 428-435.

DRUMMOND, I.A., MAJUMDAR, A., HENTSCHEL, H., ELGER, M., SOLNICAKREZEL, L., SCHIER, A.F., NEUHAUSS, S.C., STEMPLE, D.L., ZWARTKRUIS, F., RANGINI, Z. et al. (1998). Early development of the zebrafish pronephros and analysis of mutations affecting pronephric function. Development 125: 4655-4667.

HORSFIELD, J., RAMACHANDRAN, A., REUTER, K., LAVALLIE, E., COLLINSRACIE, L., CROSIER, K. and CROSIER, P. (2002). Cadherin-17 is required to maintain pronephric duct integrity during zebrafish development. Mech. Dev. 115: 15-26.

HYNES, R.O. and LANDER, A.D. (1992). Contact and adhesive specificities in the associations, migrations and targeting of cells and axons. Cel/68: 303-322.

INOUE, T., CHISAKA, O., MATSUNAMI, H. and TAKEICHI, M. (1997). Cadherin6 expression transiently delineates specific rhombomeres, other neural tube subdivisions and neural crest subpopulations in mouse embryos. Dev. Biol. 183: 183-194.

INOUE, T., TANAKA, T., SUZUKI, S.C. and TAKEICHI, M. (1998). Cadherin-6 in the developing mouse brain: Expression along restricted connection systems and synaptic localization suggest a potential role in neuronal circuitry. Dev. Dyn. 211: 338-351.

KIMMEL, C.B., BALLARD, W.W., KIMMEL, S.R., ULLMANN, B. and SCHILLING, T.F. (1995). Stages of embryonic development of the zebrafish. Dev. Dyn. 203: 253-310.

KREIDBERG, J.A., SARIOLA, H., LORING, J.M., MAEDA, M., PELLETIER, J., HOUSMAN, D. and JAENISCH, R. (1993). $W t-1$ is required for early kidney development. Cel/74: 679-691.

LIU, Q., LIU, B., WILSON, A.L. and ROSTEDT, J. (2006). Cadherin-6 message expression in the nervous system of developing zebrafish. Dev. Dyn. 235: 272278.

MAH, S.P., SAUERESSIG, H., GOULDING, M., KINTNER, C. and DRESSLER, G.R. (2000). Kidney development in cadherin-6 mutants: Delayed mesenchyme-to-epithelial conversion and loss of nephrons. Dev. Biol. 223: 38-53.

MURAKAMI, T., HIJIKATA, T., MATSUKAWA, M., ISHIKAWA, H. and YORIFUJI, H. (2006). Zebrafish protocadherin 10 is involved in paraxial mesoderm development and somitogenesis. Dev. Dyn. 235: 506-514.

NAKAGAWA, S. and TAKEICHI, M. (1998). Neural crest emigration from the neural tube depends on regulated cadherin expression. Development125:2963-2971. 
REDIES, C. (1995). Cadherin expression in the developing vertebrate CNS: From neuromeres to brain nuclei and neural circuits. Exp. Cell Res. 220: 243-256.

SERLUCA, F.C. and FISHMAN, M.C. (2001). Pre-pattern in the pronephric kidney field of zebrafish. Development 128: 2233-2241.

TAKEICHI, M. (1991). Cadherin cell adhesion receptors as a morphogenetic regulator. Science 251: 1451-1455.

TORRES, M., GOMEZ-PARDO, E., DRESSLER, G.R. and GRUSS, P. (1995). Pax2 controls multiple steps of urogenital development. Development 121: 40574065.

VIZE, P.D., SEUFERT, D.W., CARROLL, T.J. and WALLINGFORD, J.B. (1997). Model systems for the study of kidney development: Use of the pronephros in the analysis of organ induction and patterning. Dev. Biol. 188: 189-204.

WEINBERG, E.S., ALLENDE, M.L., KELLY, C.S., ABDELHAMID, A., MURAKAMI, T., ANDERMANN, P., DOERRE, O.G., GRUNWALD, D.J. and RIGGLEMAN, B. (1996). Developmental regulation of zebrafish MyoD in wild-type, no tail and spadetailembryos. Development 122: 271-280.
WESTERFIELD, M. (1995). The Zebrafish Book: a guide for the laboratory use of zebrafish (Danio rerio). Eugene, OR: University of Oregon Press.

XIANG, Y.Y., TANAKA, M., SUZUKI, M., IGARASHI, H., KIYOKAWA, E., NAITO, Y., OHTAWARA, Y., SHEN, Q., SUGIMURA, H. and KINO, I. (1994). Isolation of complementary DNA encoding K-cadherin, a novel rat cadherin preferentially expressed in fetal kidney and kidney carcinoma. Cancer Res. 54: 3034-3041.

YAGI, T. and TAKEICHI, M. (2000). Cadherin superfamily genes: Functions, genomic organization and neurologic diversity. Genes Dev. 14: 1169-1180.

Received: 11th July 2006 Reviewed by Referees: 19th October 2006 Modified by Authors and Accepted for Publication: 19th December 2006 Published Online: 17th January 2007 